\title{
2020 Southern Regional Meeting
}

\section{AWARD RECIPIENTS}

\section{AFMR Henry Christian Award}

Huixian Hong, University of Alabama at Birmingham

Priyadarshini Loganathan, Texas Tech University Health Sciences Center

Majd Michael, Texas Tech University of El Paso

Cardiovascular Case Report Award

Drew Daniels, University of Mississippi Medical Center

Ahmad Hallak, Texas Tech University

Christen H. Harris, LSU Health Sciences Center

James N. Kiage, University of Tennessee Health Science Center

Haneen Mallah, Texas Tech University Health sciences Center

Michael B. Omar, University of Florida College of Medicine

Parikshat Sharma, Golden State Hospitalists

Richard D. Silva-Cantillo, VA Caribbean Healthcare

Nathan Solomon \& Irene Oransky-Solomon Award

Caleb Max, University of Tennessee Health Science Center

Ashkan Shahbandi, Tulane University School of Medicine

SAFMR/SSCI Junior Faculty Research Travel Award

Harsha Karanchi, Medical University of South Carolina

Tadashi Yoshida, Tulane University

\section{SAFMR/SSCI Student Research Award}

Ashwin Ajith, Georgia Cancer Center

Annie L. Bell, Tulane University School of Medicine

Abraham B. Chabot, Tulane University School of Medicine

Caroline G. Coleman, Emory University School of Medicine

Clayton L. Davis, University of Arkansas Medical Sciences

Heather Davis, Ochsner Clinic Foundation

Travis Dobbin, University of Queensland/Ochsner Clinical School

Melissa R. Downey, Medical College of Georgia

Emily E. Evans, Emory University

Anam Haque, McGovern Medical School

Kristin Harrington, Rollins School of Public Health, Emory University School of Medicine

Carl Haupt, Ochsner Health System

Candace M. Howard, University of Mississippi Medical Center

Jeanne Ishimwe, University of Mississippi Medical Center

Lauren A .Jeffers, Emory University

Rebecca Joseph, University of Central Florida

Chin Fung Kelvin Kan, Ochsner Clinical School-University of Queensland, Laboratory of Translational Cancer Research

Mary Kelly, Emory University

Darya Khani, Emory University 
Jae Gyun Maeng, Quillen College of Medicine - East Tennessee State University

Ganesh B. Maniam, Texas Tech University Health Science Center

Will McDonough, University of South Alabama

Sarah Miller, University of Mississippi Medical Center

Emily N. Nguyen, Texas Tech University Health Sciences Center

Kailash N. Pandey, Tulane University Health Sciences Center

Jay R. Patel, University of Tennessee Health Sciences Center

Pranav Prabu, Medical College of Georgia

Owen Richfield, Tulane University

Faaiz Saad, University of Alabama at Birmingham

Darius Stephens, Morehouse School of Medicine

Seth Swinney, Texas Tech University Health Science Center

Thomas S. Truong, Ochsner Health System

Sidney Tucker, University of Tennessee Health Science Center

Vipin Varghese, University of Queensland - Ochsner Clinical School

Elliot Varney, University of Mississippi Medical Center

Hannah Wilkerson, Texas Tech University Health Sciences Center

Kenyada Williams, Meharry Medical College

Di Xia, Augusta University

George S. Yeung, Ochsner Clinical School, University of Queensland

Katherine Yu, McGovern Medical School

SAFMR/SSCI Trainee Research Award

Max Adelman, Emory University

Hiba Ali, Ochsner Medical Center

Genanew D. Bedanie, Texas Tech University Health Science Center

Anil Chimakurthy, Louisiana State University Health Sciences Center

Craig Cook, Emory University

Patrick Dauphin, University of Mississippi Medical Center

Omar Elfeky, Louisiana State University Health Sciences Center

Vincent Gacad, Tulane University

Akshay Goel, University of Arkansas for Medical Sciences

Robert A. Greer, University of Mississippi Medical Center

Ibrahim Haddad, East Tennessee State University

Kirstin G. Hesterberg, University of Tennessee Health Science Center

Ilya Ivyanskiy, Texas Tech University HSC Amarillo

Isaac Jaben, Medical University of South Carolina

Sanjida Jahan, Ochsner Clinic Foundation

Sarah M. Jeong, University of Mississippi Medical Center

Daniel Kelly, University of Mississippi Medical Center

Varun Kohli, East Tennessee State University

Katherine Mascia, University of Alabama at Birmingham

Dexter Matrana, Louisiana State University Health Sciences Center

Lara McKean Baste, Ochsner Medical Center

Ryley McPeters, Louisiana State University Health Sciences Center

Neal Mehta, White River Health System

Taylor Morris, Ochsner Clinic Foundation

Yara Mouawad, Tulane University

Amelia Muniz Hernandez, Emory University School of Medicine

Jose E. Najul, Ochsner Clinic Foundation

Angela Nakahara, Ochsner Medical Center

Zohra Prasla, Emory University School of Medicine, Atlanta VAHCS

Nikita L. Rabelo-Pagan, University of Puerto Rico

Joel Raja, University of Tennessee Health Science Center

Kevin Rechcigl, University of Florida College of Medicine

Keerthi Reddy, University of Mississippi Medical Center

Sumana Reddy, University of Mississippi Medical Center

Arya Mariam Roy, University of Arkansas for Medical Sciences

Nicoleta Rus, Texas Tech University HSC Amarillo

Firas Safa, Tulane University 
Ayesha Sajiad, Texas Tech University Health Sciences Center

Michael Y. Soliman, Louisiana State University Health Sciences Center

Stephen J. Soufleris, University of Tennessee Health Science Center

Frederique St-Pierre, Mayo Clinic

Aditi V. Varma, Louisiana State University Health Sciences Center

Cameron B. Wilhoit, Medical University of South Carolina

Jesse Juncong Xie, University of Arkansas for Medical Sciences

John Xie, Tulane Medical Center

SSCI Nephrology Young Investigator Scholar Award

Fabian Bock, Vanderbilt University Medical Center

Hannah Gardner, University of Alabama at Birmingham

Cesar F. Hernandez-Arroyo, Ochsner Clinic Foundation

Arun Rajasekaran, University of Alabama at Birmingham

Matthew Sinclair, Duke University Medical Center, Duke Clinical Research Institute

Justin Sprick, Emory University, Department of Veterans Affairs Health Care System

Rohit Upadhyay, Tulane University School of Medicine

SSCI Poster Award Finalists

Edward Florez, University of Mississippi Medical Center

Lauren Jeffers, Emory University

Elliot Varney, University of Mississippi Medical Center

SSCI Young Investigator Award

Jennifer Hong, Tulane University School of Medicine

Alexander C. Razavi, Tulane University School of Medicine and Public Health and Tropical Medicine

Maria Soledad Rivera, University of Queensland, Ochsner Clinic Foundation

SSCI Young Investigator for General Medicine

Bryant Shuey, University of South Florida

SSPR Basic Science Young Investigator Award Finalists

David Box, University of Oklahoma

John I. Coon, University of Texas Medical Branch

Amy Freeman, University of Alabama at Birmingham

SSPR Clinical Science Young Investigator Award Finalists

Leslie-Anne Dietrich, UT Health Science San Antonio

Rebekah C. Gardea, University of Texas Health Science Center San Antonio

Tavleen Sandhu, University of Oklahoma

SSPR David Oelberg Honorary Trainee Travel Award

Nilesh Dankhara, University of Mississippi

Ahmed L. Elsaie, Baylor College of Medicine

SSPR Trainee Travel Award

Murad Almasri, University of Texas Medical Branch

Maria E. Barbian, Emory University

Taylor M. Boles, Rice University

Charlie Boyle, University of Tennessee Health Science Center

Gabriel Castano, University of Texas Health Science Center at San Antonio

Janhavi Govande, Texas Children's Hospital

Melissa D. Gruver, University of Tennessee Health Science Center

Morghan Jameson, ETSU Quillen College of Medicine

Maggie L. Jerome, University of Alabama at Birmingham

Mounica Konjeti, University of Tennessee Health Science Center

Marco Loayza, University of Mississippi Medical Center

Mishika Malik, University of Connecticut School of Medicine, Connecticut Children's 


\section{Abstracts}

Radhika Mohan, UT Health Science Center at San Antonio

Ann E. Murphy, University of Tennessee Health Science Center Anshu A. Paul, Baylor College of Medicine

Morgan J. Sims, Medical University of South Carolina

Ellen P. Soufleris, University of Tennessee Health Science Center

Mark Stephens, University of Kentucky

Bianca M. Vamesu, University of Alabama at Birmingham

Sean Woods, Emory University

SSPR Young Faculty Travel Award

Nicholas Carr, Brooke Army Medical Center

Eric B. Ortigoza, UT Southwestern Medical Center

Mitali Pakvasa, Emory University, Children's Healthcare of Atlanta 